\title{
Characterization of Al-8090 superplastic materials using orientation imaging microscopy
}

\author{
H. Garmestani ${ }^{\text {a,* }}$, P. Kalu ${ }^{\text {a }}$, D. Dingley ${ }^{1, b}$ \\ ${ }^{a}$ FAMU/FSU College of Engineering, Department of Mechanical Engineering and Center for Materials Research and Technology (MARTECH), \\ Tallahassee, FL 32317, USA \\ b Physics Department, Bristol University, Bristol BS8 ITL, UK
}

Received 30 July 1996; received in revised form 19 June 1997

\begin{abstract}
A fundamental understanding of the deformation mechanisms of superplasticity requires a detailed characterization of the microstructure. For certain studies, optical microscopy, transmission electron microscopy (TEM) and scanning electron microscopy (SEM) techniques used in conventional mode may be inadequate for detailed analysis. This paper presents the use of a newly developed technique, 'orientation imaging microscopy' (OIM) in characterizing the microstructure of an Al-8090 alloy deformed in uniaxial tension to strains of 15,70 and $660 \%$ at $520^{\circ} \mathrm{C}$ and $5 \times 10^{-4} \mathrm{~s}^{-1}$ strain rate. In OIM the microstructure is constructed from the measured crystal orientations obtained from points on the specimen surface distributed in a hexagonal grid. Neighboring measurements with a misorientation greater than a specified value, $\omega$, misorientation angle criteria (designated by the researcher) are deemed to define the location of grain boundaries. These boundary lines can be interpreted as high angle grain boundaries or subgrain boundaries depending on the value of $\omega$. The implications of the analyses on superplastic deformation are discussed. (C) 1998 Elsevier Science S.A.
\end{abstract}

Keywords: Orientation imaging microscopy; Superplastic materials; Microstructure

\section{Introduction}

Superplasticity refers to the ability of some materials to deform to exceptionally large strains at elevated temperatures. In this process elongation's as high as $4850 \%$ have been recorded [1]. Current understanding of superplasticity and mechanisms of superplastic forming indicates that grain size and grain shape are of fundamental importance. Such analysis has been based on traditional methods of microstructural characterization including conventional optical, scanning electron microscopy (SEM) and transmission electron microscopy (TEM) in addition to standard X-ray texture measurements [2]. The SEM technique, used in the conventional method, provides similar information to an optical microscope albeit at much higher resolution and magnification and depends on etching techniques

\footnotetext{
* Corresponding author. Tel.: + 1904 6445993; fax: + 1904 6441227; e-mail: garm@magnet.fsu.edu

${ }^{1}$ Present address: TSL, Provo, UT, 84604, USA.
}

to reveal grain boundaries. The TEM analysis of grain structure and subgrain formation provides both morphological and crystallographic data but can only provide such information for a few individual grains or at best a small region of the microstructure. Such analyses do not provide an adequate global picture, and can result in erroneous conclusions. The X-ray technique provides texture data averaged over a large area with no information with respect to spatial distribution of the grain orientations or spatially specific grain misorientations.

Studies employing the above named techniques have shown that the ideal microstructure for superplastic deformation consists of small $(\leq 10 \mu \mathrm{m})$ equiaxed grains. During superplastic deformation any initial grain alignment of the microstructure will disappear and a gradual increase in grain size may occur at the later stages of deformation [3,4]. These works have demonstrated that grain boundary sliding is the main mechanism of superplastic deformation [5] and that this is accommodated by slip or diffusion creep [6]. It has 
been suggested that grain size, grain morphology and grain boundary misorientation are important parameters for grain boundary sliding, although there exists some controversy as to the optimum value of grain boundary misorientation necessary for grain boundary sliding during superplastic deformation [7,8]. It follows that in addition to standard microstructural characterization, grain-to-grain misorientation studies are crucial in understanding the deformation mechanisms of such materials. Grain-to-grain misorientation measurements can be obtained by analyzing spot and Kikuchi patterns observed in the TEM or from channeling diffraction patterns and electron backscattering patterns observed in SEM studies. However, in order to obtain detailed information of high statistical reliability that will account for a global picture of the superplastic microstructure crystal orientation measurements from large areas of the samples have to be analyzed. Manual indexing of TEM and SEM patterns for such large surveys is impractical. However, with the advent of automatic indexing of electron backscattered pattern (EBSP), the required large number of orientation measurements and grain-to-grain misorientation measurements can be obtained. The automated EBSP technique has been extended to include mapping of the crystal orientations and is referred to as orientation imaging microscopy (OIM).

This paper provides the results of a detailed microstructural characterization of a superplastically deformed Al-8090 material using OIM. The technique has enabled the crystallographic features and the morphology of the microstructure to be depicted over large areas of the sample.

\section{Justification for orientation imaging microscopy}

In conventional optical microscopy, grains and grain boundaries are revealed by chemical etching of the specimen surface. This technique may give a false impression of the microstructure since it is impossible to distinguish small from large misorientation differences amongst the grains. For instance both slight and large changes in the misorientation angle between adjacent grains can produce significant changes in shade. Also, grain boundary etchings which are supposed to be sensitive to residual strain or energy gradients at the boundary, in practice may not be so, because solute segregation and precipitation may mask the etching differential expected. Hence, low angles may not be distinguished from high angle boundaries. It is thus uncertain whether the true microstructure or what particular aspects of that microstructure may have been revealed. SEM, used in both conventional secondary electron imaging mode and in the channeling contrast mode, provides similar information to the optical mi- croscope though at much higher resolution. It is difficult to discern the lattice orientation of individual grains, and as such the essential crystallographic information is missing.

In TEM, the diffraction contrast image shows all changes in orientation and hence distinguishes each grain and the grain boundaries that separate them. However, quantification of the microstructure in terms of types of grain boundary requires that electron diffraction patterns be obtained from each area. Even distinguishing low angle from high angle boundaries can not be done from examination of the conventional image alone. A simple tilt boundary of $2^{\circ}$ misorientation has edge dislocations in it, spaced $7.2 \mathrm{~nm}$ apart. The overlapping strain field from each dislocation prohibits resolving them individually and, consequently, any chance of recognizing the boundary for what it is. Furthermore, TEM analysis of grain and subgrain structures can only provide information from a small region of the microstructure. Such analyses may not provide an adequate global picture of the microstructure and can result in erroneous conclusions.

X-ray diffraction technique, on the other hand, though providing average texture data from a large area neither provides the important information on the spatial distribution nor interrelation of individual grains and subgrains.

Following up on the work of Venables [9], who developed the EBSP technique in the SEM, Dingley [10] developed a computer assisted method for on-line analysis of diffraction patterns. Adams et al. [11] extended this to enable full automation of the technique. These diffraction patterns are formed in the same manner as Kikuchi patterns in the TEM, but result from backscattering of electrons out of the top surface of the sample. Hence they can be obtained from bulk samples. The specimen is inclined in the SEM at $70^{\circ}$ to the incident electron beam. The diffraction patterns are imaged on a phosphor screen placed close to it, as illustrated in Fig. 1. The phosphor screen is viewed

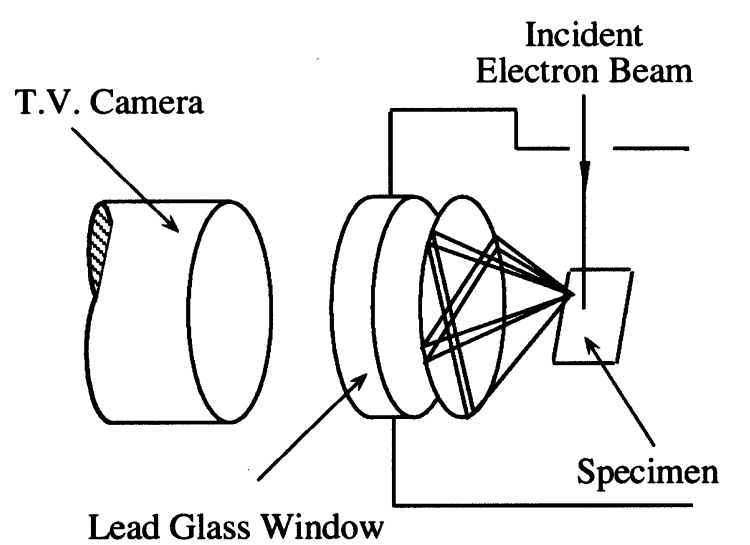

Fig. 1. Schematics of BKD technique. 


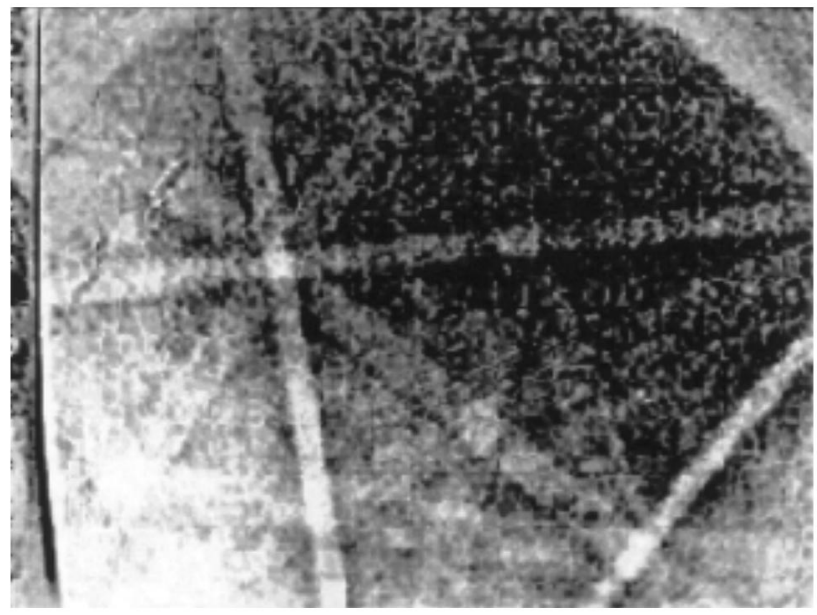

Fig. 2. Typical Kikuchi pattern obtained by employing EBSP technique for an $\mathrm{Al}-\mathrm{Li}$ specimen.

through an optical port using a high gain television camera which in turn is interfaced to a computer. The resulting Kikuchi pattern (Fig. 2) is recorded in the computer and indexed. By indexing successive patterns from hundreds of selected points on the sample surface, sufficient data can be collected to determine both macroscopic and local orientation texture, and as such can provide a detailed survey of nearest neighbor orientation relationships.

Automation of the EBSP technique [12,13] through OIM opened a new perspective in materials characterization which previously had only existed using TEM for thin foil specimens. In OIM, the EBSPs are collected from points on the sample surfaces and distributed over a hexagonal grid. The patterns are automatically indexed, and the gradients of the local lattice orientation in the material is obtained. From this data, a grain boundary map can be constructed. This map is essentially a representation of the microstructure, and reveals the changes in crystal orientation over the specimen surface. The boundary contrasts on the map are formed at regions which satisfy a misorientation angle criteria, $\omega$, input by the investigator. In other words, a boundary is deemed to exist when the misorientation between two neighboring points on the hexagonal grid exceeds a user defined misorientation $\omega$.

The difference between orientation and what is commonly referred to as a 'misorientation' here is the choice of reference axes. For misorientation, the reference frame is taken on the reference axes of one of the two grains. If the absolute orientation of each of the two grains are represented by a matrix of cosine angles with respect to a laboratory frame (orientation) designated $\mathbf{g}_{1}$ and $\mathbf{g}_{2}$ respectively, the misorientation $\mathbf{g}_{12}=\mathbf{g}_{1}^{-1} \mathbf{g}_{2}$ is the matrix which represents the rotation of $\mathbf{g}_{1}$ into $\mathbf{g}_{2}$. This rotation matrix can also be represented in terms of the Euler angler $\left(\phi_{1}, \varphi, \phi_{2}\right)$. It can alternatively be represented in terms of a rotation of $\theta$ about a specific axis $d$ where $d$ is a direction common to both the reference coordinate system and the sample coordinate system. This angle/axis type of representation is more usually associated with grain misorientation to define special type boundaries: low angle, high angle, twin, and coincident site lattices (CSLs) [14]. Such a definition is used here to identify the grain boundaries and to measure misorientation based on one angle. This representation is common in materials science community by only considering the misorientation angle and totally ignoring the common axis of rotation between the two grains, or across a boundary of interest in a microstructure.

\section{Material and experimental}

The material used in this study was an $8090 \mathrm{Al}-\mathrm{Li}$ alloy of nominal composition $2.39 \mathrm{Al}-1.21 \mathrm{Li}-0.64 \mathrm{Cu}-$ $0.12 \mathrm{Mg}$ (in wt.\%). Tensile coupons were prepared with gage dimensions of $0.5 \times 0.25 \times 0.1$ in. (in length, width, and thickness respectively) and having the tensile axis parallel to the rolling direction. The coupons were superplastically deformed to different strains at $520^{\circ} \mathrm{C}$ (and air cooled) using a nominal strain rate of $5 \times 10^{-4} \mathrm{~s}^{-1}$. Samples for analyses were sectioned from the gage sections of the coupon, and electropolished following an initial sequence of mechanical polishing.

OIM analysis was performed on specimens deformed to 15,70 and $660 \%$. This involved automatic generation and indexing of several thousand EBSP measurements taken on a hexagonal grid of points, with spacing of about $2.5 \mu \mathrm{m}$ at each step. The image of the microstructure was reconstructed by creating grain boundary maps from the EBSP measurements. Designation of grain boundaries was based on a boundary criteria, $\omega$, provided by the researcher. Misorientation angle, $\theta$, is calculated for every point in the microstructure and then compared with $\omega$. In this paper, two criteria, $1^{\circ}<\omega<10^{\circ}$ and $\omega \geq 10^{\circ}$ were considered. By employing these criteria during the generation of the grain boundary maps, different images of the microstructure were constructed. Also, the image quality (IQ) of the measured EBSPs represented as gray scale was used to produce an IQ micrograph where the gray level shade was proportional to the IQ value. In this representation, black points equal to an IQ of 0, while white represents an IQ of 27. 


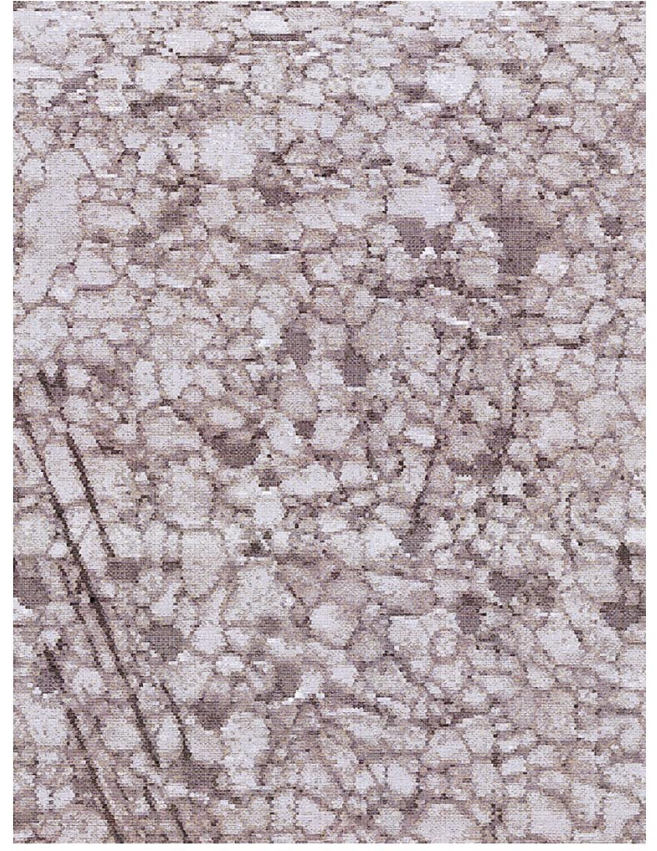

- $10.0 \mu \mathrm{m}-10$ steps boundary levels: shaded $1 Q 21 \ldots 229$ Fig. 3

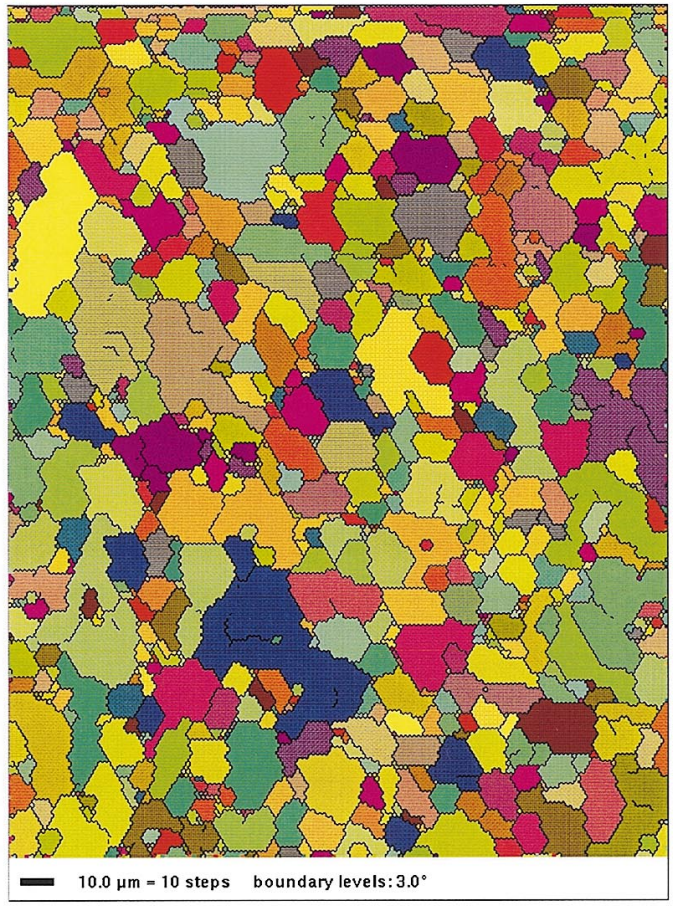

(a)

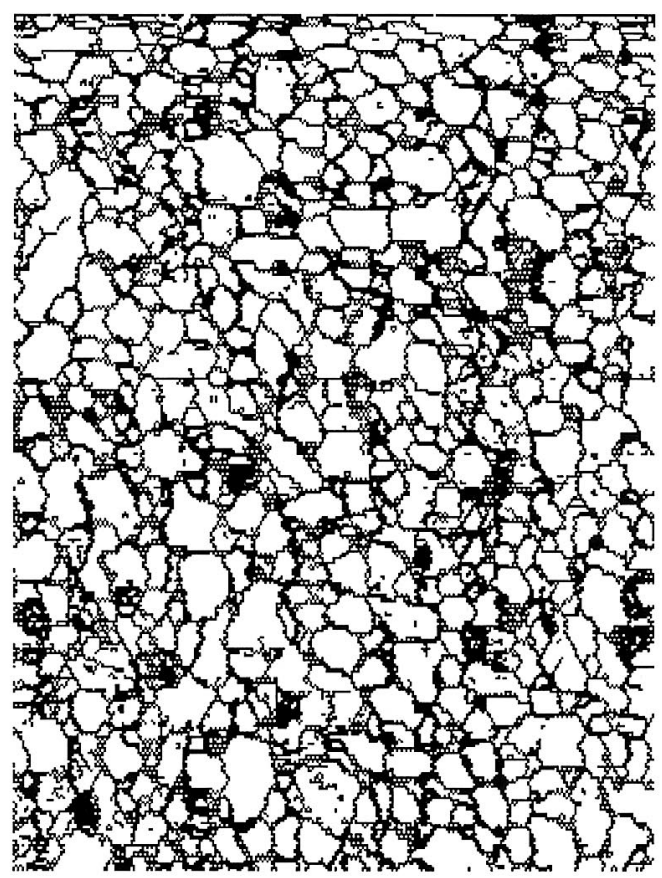

- $10.0 \mu \mathrm{m}-10$ steps boundary levels: $1.0^{\circ} 10^{\circ}$ Fig. 4

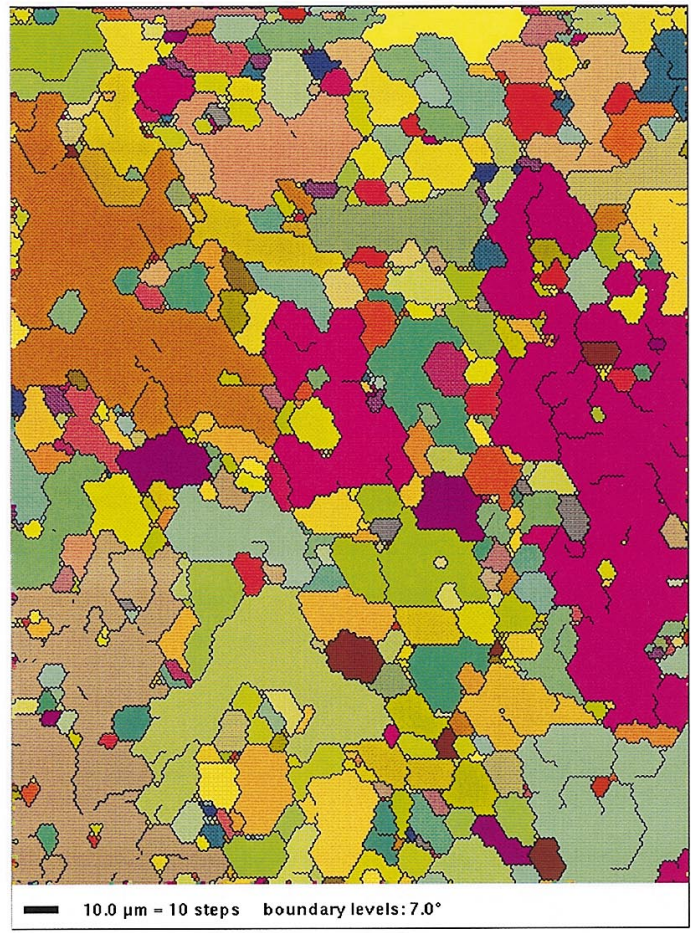

(b)

Fig. 5

Fig. 3. An image quality micrograph for a $15 \%$ deformed SPF sample of Al-8090 material obtained from the OIM technique.

Fig. 4. An OIM micrograph with thick lines representing high angle grain boundaries $\left(>10^{\circ}\right)$ and thinner black lines depicting low angle grain boundaries.

Fig. 5. Misorientation boundary micrographs. Black lines depict boundaries with misorientations greater than (a) $3^{\circ}$ and (b) $7^{\circ}$. 


\section{Results and discussion}

A typical IQ micrograph of the measured EBSPs for the sample deformed to $15 \%$ is presented in Fig. 3. There is no quantitative information on the grain boundaries in this micrograph: the darker regions represent locations in the microstructure where the IQ is low, i.e. where poor quality EBSPs were obtained. Fig. 4 is a grain boundary map constructed using the same data set as in Fig. 3, except that it is now drawn with boundary criteria of $1^{\circ}<\omega<10^{\circ}$ for thin lines and $\omega \geq 10^{\circ}$ for thick lines. In this case, boundaries with misorientation between $1^{\circ}$ and $10^{\circ}$ are revealed as thin lines, and those boundaries with misorientation above $10^{\circ}$ are revealed as thick lines. If both the thin and thick lines are equally perceived as grain boundaries, the microstructure is seen to be essentially equiaxed. In both Figs. 3 and 4 , the average grain size is $\approx 10 \mu \mathrm{m}$ in diameter. Fig. 5(a) and (b) are other grain boundary maps constructed from the same data set as Fig. 4. Here, two different boundary criteria, (a) $\omega \geq 3^{\circ}$, and (b) $\omega \geq 7^{\circ}$ were employed. This means that only boundaries with misorientations greater than $3^{\circ}$ in case of Fig. 5(a), and misorientations greater than $7^{\circ}$ in case of Fig. 5(b) are shown. In Fig. 6 the grain boundary map corresponding to the boundary criteria $\omega \geq 15^{\circ}$ is shown. In the latter image it appears that the microstructure consists of clusters of fine grains sandwiched between two large grains ' $\mathrm{A}$ ' and ' $\mathrm{B}$ '. The EBSP pole figures obtained from the grains ' $A$ ', and 'B' (see Fig. 7) showed that the microstructure exhibited a [100] type texture, with two strong units ' $a$ ', and ' $b$ '. Analysis of the data demonstrated that the poles ' $a$ ' and ' $b$ ' were produced by the corresponding segments ('A' and 'B', respectively) of the microstructure noted in Fig. 6. Segments ' $A$ ' and ' $B$ ' had similar orientation but rotated $30^{\circ}$ about [001] relative to each other. Each of these grains contain small subgrains. It is important to note that the fine grain regions sandwiched between grains ' $A$ ' and ' $B$ ' had random orientation. A similar result was obtained when a boundary criteria of $\omega \geq$ $10^{\circ}$ was used.

The grain boundary maps of the specimen deformed to $70 \%$ are shown in Fig. 8(a) and (b). There has been some removal at grain boundaries of data of low confidence in the accuracy of the indexing. This procedure replaces a low confidence index point in the orientation of that of its neighbor. It results in a slightly artificial appearance in the shapes of the grains. Fig. 8(a) shows the microstructure constructed based on the boundary criteria $1^{\circ}<\omega<10^{\circ}$ for thin lines and $\omega \geq$ $10^{\circ}$ for thick lines. The structure appeared equiaxed and similar to that observed when a comparable grain boundary criteria was used to analyze the microstruc- ture of the $15 \%$ deformed sample. Fig. 8(b) presents another grain boundary map drawn with boundary criteria, $\omega \geq 10^{\circ}$. The visual impression is now quite different - no longer equiaxed. The corresponding pole figures for this region is presented in Fig. 9 and shows a tendency towards grain randomization.

At the termination of superplastic forming, $660 \%$, a significant amount of grain growth was observed (Fig. 10(a)-(c)). Fig. 10(a) shows the IQ micrograph without reference to grain boundary misorientation. In Fig. 10(b), thin and thick lines are drawn based on the boundary criteria $1^{\circ}<\omega<10^{\circ}$ and $\omega \geq 10^{\circ}$ respectively. It is seen that very few low angle boundaries exist in Fig. 10(b), suggestive that the microstructure consists essentially of high angle boundaries. This fact was made clearer when a single boundary criteria of $\omega \geq 10^{\circ}$ was used, as shown in Fig. 10(c). The structure consists of coarse grains and very similar to that obtained in Fig. 10(b). The grain size is almost 200\% larger than that observed in the corresponding OIM in Figs. 4-6 for the material deformed $15 \%$. The thickness of the grain boundaries as depicted in Fig. 9(a) indicates a high level of degradation at these interfaces. Such points indicate that the Kikuchi patterns did not form on the phosphor screen and as a result the microstructure exhibits a high level of noncrystallinity. The accumulation of such points (spots) results in the widening of the grain boundaries. This thickening process can be interpreted as increased intergranular dislocation activity. Pole figures for this level of strain indicates a high level of randomness of the microstructure.

Reference to Fig. 4(a)-(b) to Fig. 8(a)-(d) shows that up to a strain of $70 \%$, the microstructure of the material is that of large grains containing a fine internal subgrain structure of near misorientation angle less than $10^{\circ}$. In addition, in the material deformed to only $15 \%$ the large grains sandwiched strings of grains an order of magnitude smaller. These small grains misoriented by large angles. At the highest strains of $660 \%$, the microstructure had changed such that the large grains no longer contained the fine network of subgrains and strings of small grains between the larger grains no longer existed. At the same time the crystallographic texture had become more random, with the volume fraction of $\langle 100\rangle$ oriented grains decreasing substantially.

Optical micrographs of the low strained material showed an apparent equiaxed structure of grains. The etchant had clearly failed to distinguish high angle from low angle boundaries. This has important consequences with respect to the application of theories of superplastic deformation. In all such theories $[16,17]$ the relationship between strain rate and stress is formed to be a function of grain size, for example 


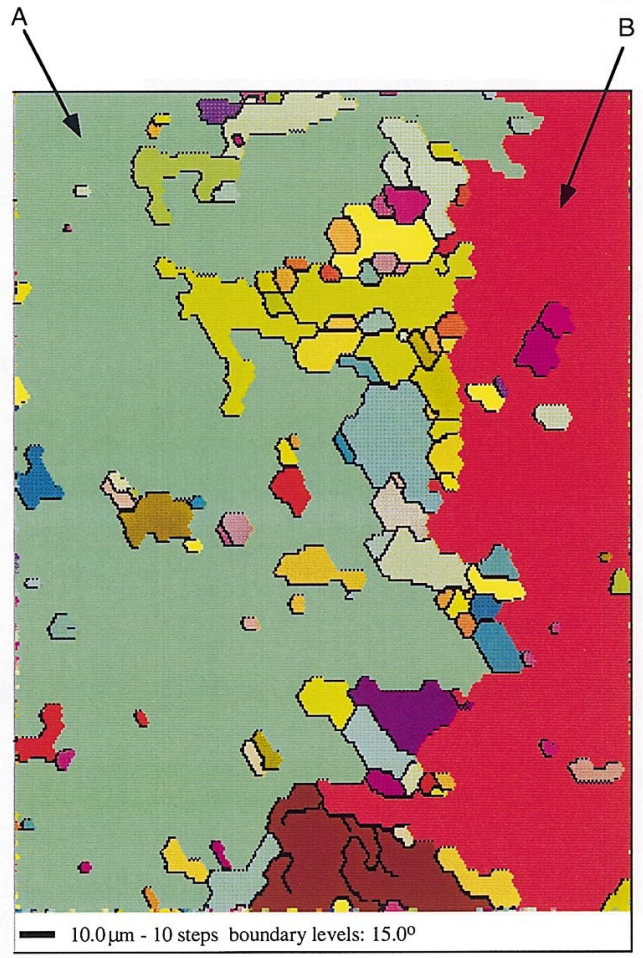

Fig.6
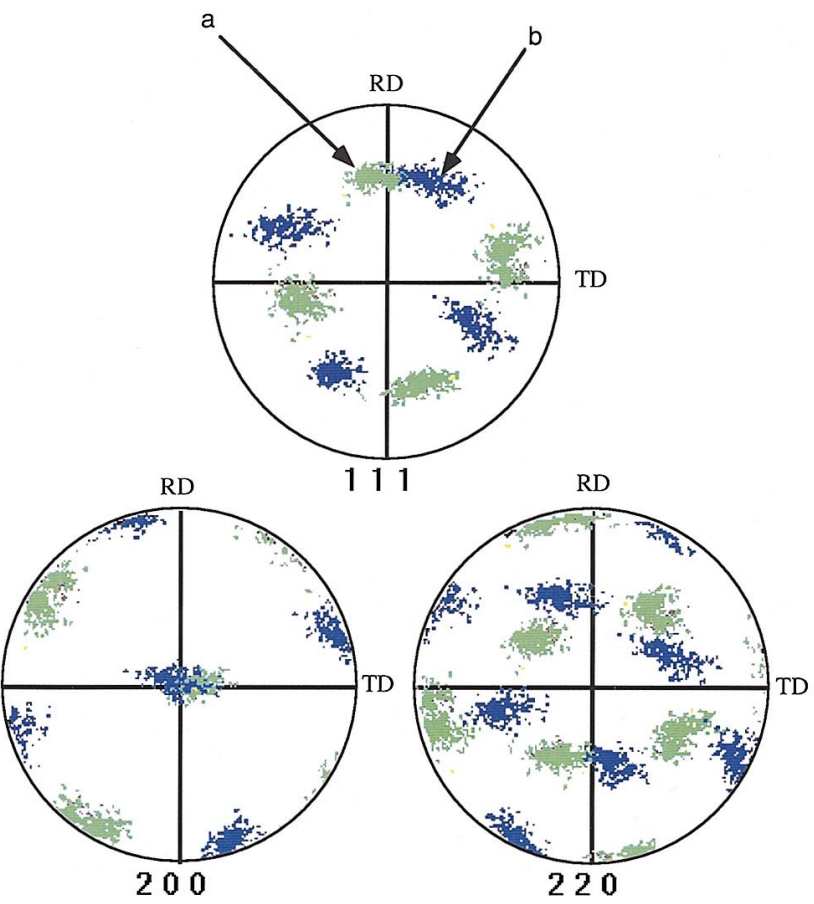

Fig.7

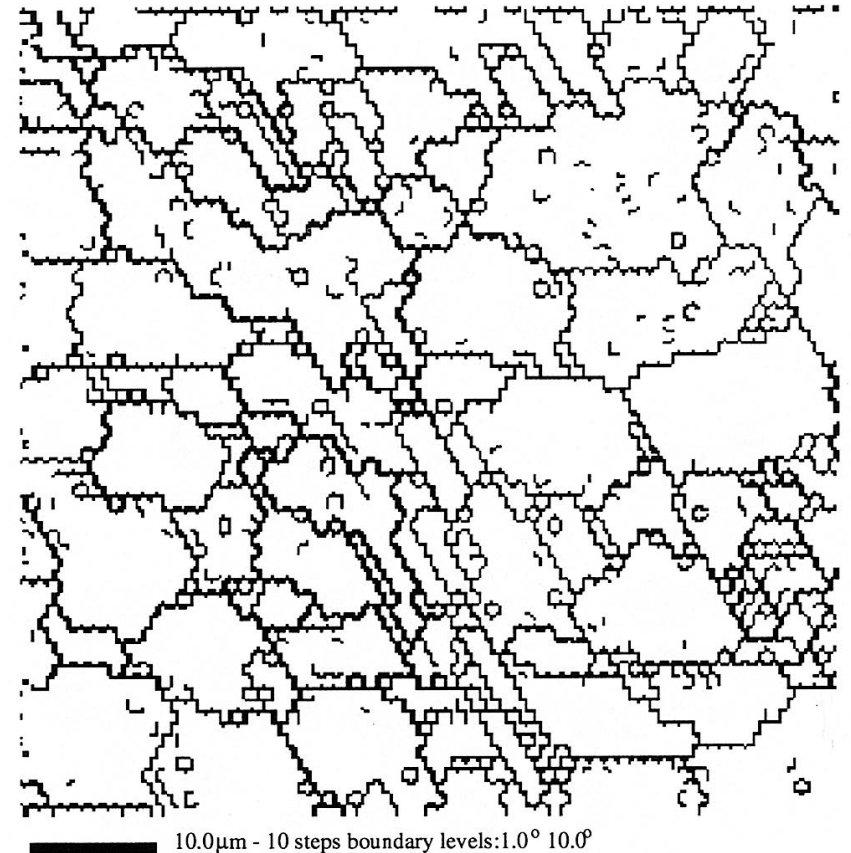

(a)

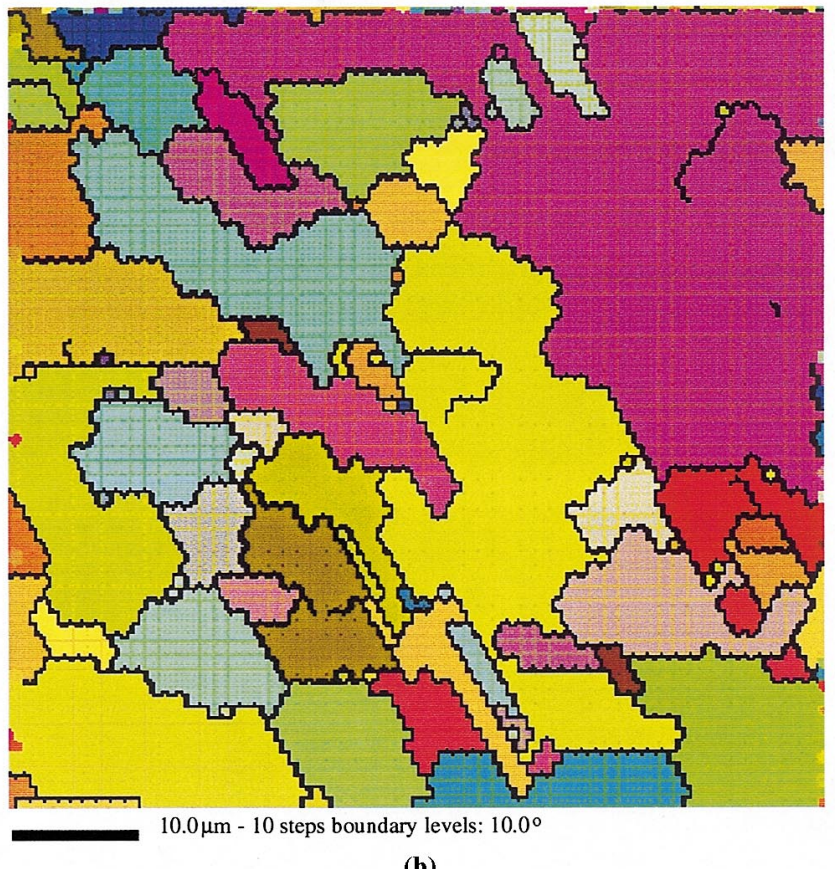

Fig. 8

Fig. 6. The grain boundary map corresponding to a boundary criteria of $\omega>15^{\circ}$ showing two large low angle grain boundary regions 'A', and 'B'.

Fig. 7. Pole figure representation of grains 'A' and 'B' shown in Fig. 6. Poles 'a' and 'b' are produced by these two large grains.

Fig. 8. The OIM micrograph for the specimen deformed to $70 \%$ elongation. (a) shows the microstructure constructed with thin lines depicting grain boundaries across which the misorientation lies between $1^{\circ}$ and $10^{\circ}$; (b) the corresponding OIM in which only the $10^{\circ}$ boundaries are drawn, is shown. 


$$
\begin{aligned}
\frac{\dot{\varepsilon} k T}{D_{\mathrm{b}} G b} \cong 2\left(\frac{b}{d}\right)^{2}\left(\frac{\sigma}{G}\right)^{2} & \text { Ball-Hutchinson Model } \\
\frac{\dot{\varepsilon} k T}{D_{\mathrm{b}} G b} \cong 100\left(\frac{b}{d}\right)^{3}\left(\frac{\sigma}{G}\right) & \text { Spring and Nix Model }
\end{aligned}
$$

Both models are dependent on grain boundary character and are both greater for high angle boundaries than low angle boundaries. Thus the value of ' $d$ ' to be used in the above equalities should be that describing the average grain size of grains surrounded by high angle boundaries (i.e. 'true grain'). The OIMs of Figs. 4-8 show that in the Al-Li alloy studied, the size and distribution of such grains is quite different from what might have been deduced from the optical micrograph alone. This study has therefore clearly pointed out the manner by which incorrect values could have been used in part in testing of equations when grain size data is collected from optical micrographs of etched specimens alone. These observations also indicate that some account has to be taken of the heterogeneity of the microstructure. Noting that the greater shear strain will be sustained by the smaller 'true grain' size fraction then at low strains only a small percentage of the microstructure fits this criterion, i.e. the small grains lying in between the large grains.

Both the grain boundary sliding rate and total shear sustained by these grains is therefore much greater than that calculated on the basis that the shear is uniformly distributed over the material. From the microstructure of the figure we can estimate the difference to be at least a factor of 10 . With increasing deformation both the texture and grain inhomogeneity decreased. We can

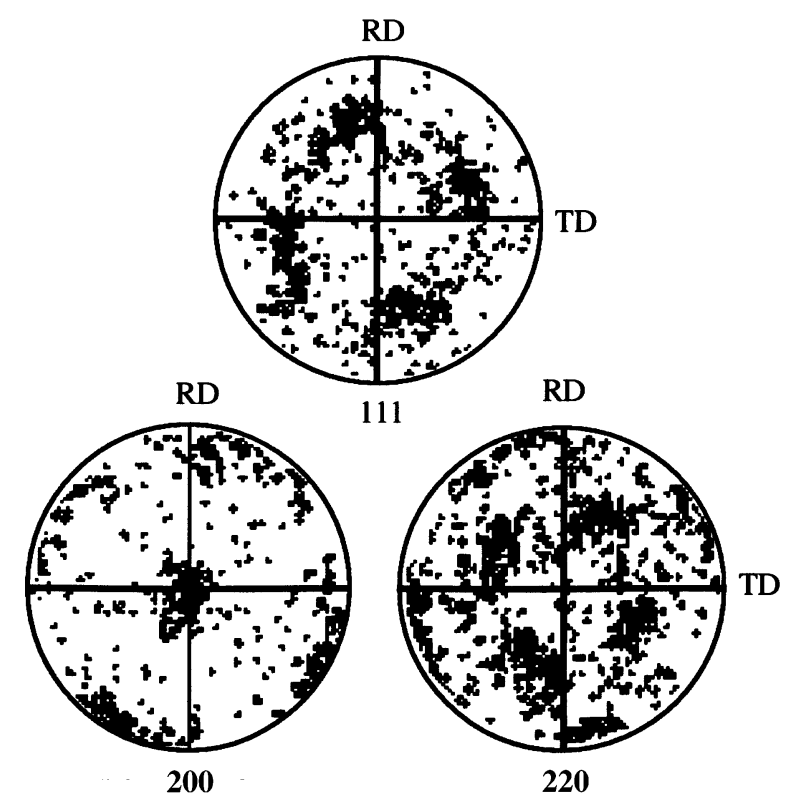

Fig. 9. The pole figure representation of the microstructure in Fig. 8. This pole figure shows a tendency towards randomization. expect therefore the application of Eqs. (1) and (2) using 'apparent' grain size to determine the value of ' $d$ ' to provide a reasonable test of the deformation models. However, we stress again that this conclusion could only be drawn because the true high deformation microstructure determined using the OIM technique, where it was seen the apparent and true microstructure were the same. In cases where the OIM technique is not used considerable caution has to be taken in the interpretation of 'apparent' microstructure.

\section{Conclusion}

This study has shown that OIM is an effective tool to investigate the evolution of microstructure in superplastic materials. The OIM technique provided an opportunity to review the range of possible interpretations that can be given to the microstructure of a Al-8090 alloy deformed to strains of 15,77 and $660 \%$. By invoking the conventional definition of high angle grain boundary, which requires a minimum misorientation of $10^{\circ}$ [15], the researchers have compared microstructures reconstructed using boundary criteria of $1^{\circ}<\omega<10^{\circ}$ and/or $\omega \geq 10^{\circ}$. The first criteria considered any boundary between $1^{\circ}<\omega<10^{\circ}$ as grain boundaries, while the second criteria recreated boundaries greater than $10^{\circ}$ as grain boundaries. The microstructures corresponding to any of the three strains can best be described as equiaxed when the boundary criteria of $1^{\circ}<\omega<10^{\circ}$ was used. This deduction did hold when a boundary criteria of $\omega \geq 10^{\circ}$ was used. At the later stage of deformation (strain of $660 \%$ ) the microstructure can be described as equiaxed. However, the early stage of deformation (strain of 15\%) showed a microstructure which consisted of clusters of fine equiaxed grains sandwiched by distinct large grains. It is evident that the results have raised some concerns on the long standing views about the grain morphology in Al-8090 alloy and its involvement in superplasticity.

In superplastic deformation, the dominant mechanism is grain boundary sliding, which is presumed to occur at regions of fine, equiaxed grains. Based on the results reported in this investigation it is reasonable to conclude that grain boundary sliding is localized in these regions of the material rather than the entire microstructure. These regions in question are the fine equiaxed grain regions, because they satisfy the microstructure prerequisite needed for grain boundary sliding.

\section{Acknowledgements}

The authors gratefully acknowledge the excellent technical assistance of D. Field of TSL. This research 

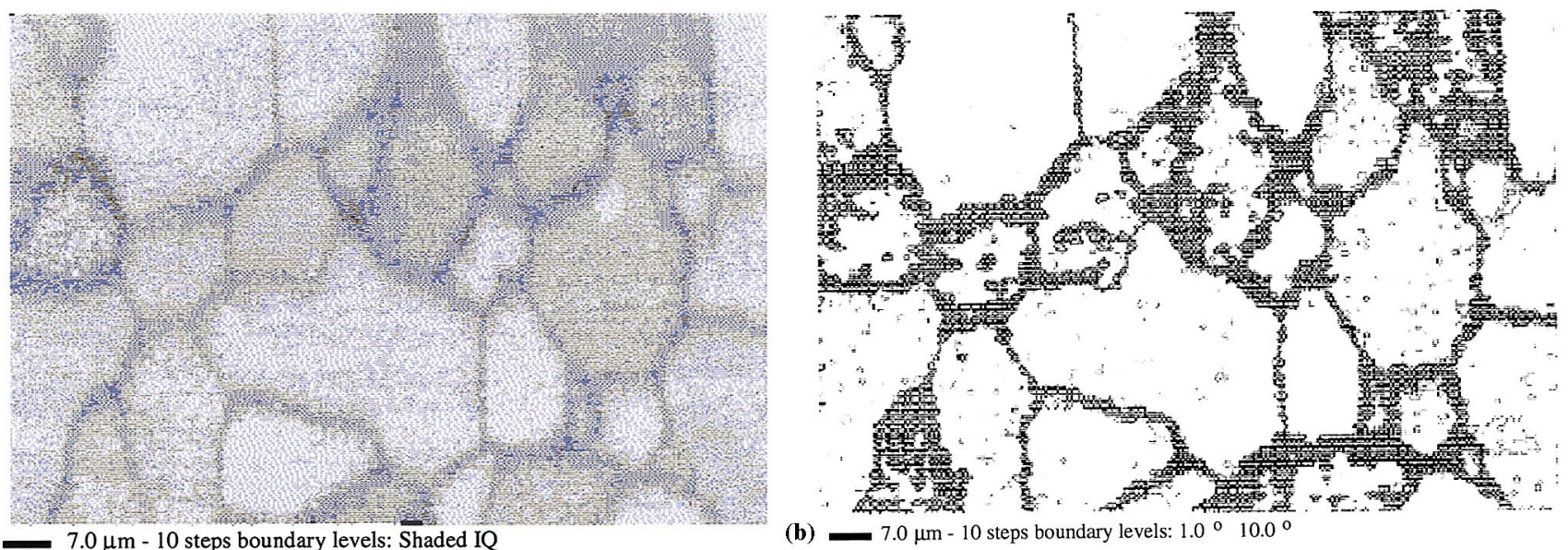

(a)

$7.0 \mu \mathrm{m}-10$ steps boundary levels: Shaded IQ

(b)

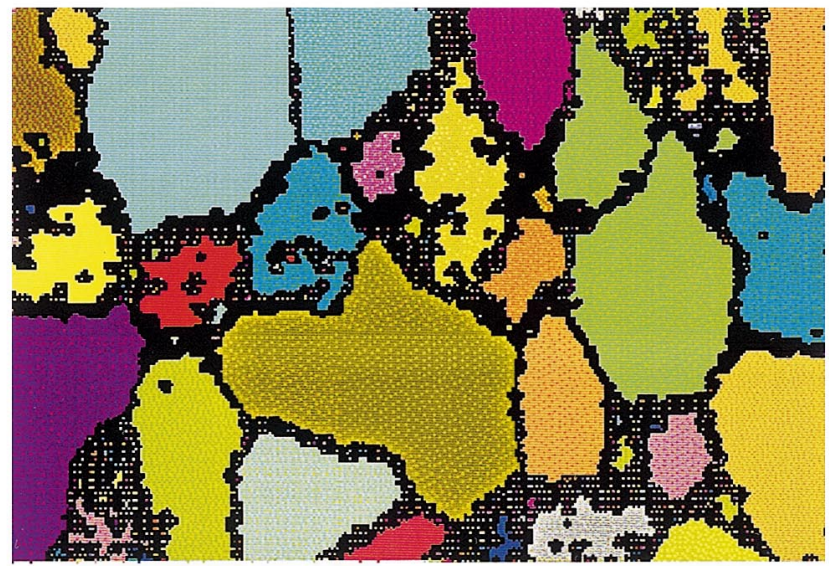

(c)

$7.0 \mu \mathrm{m}-10$ steps boundary levels: $10^{\circ}$

Fig. 10. OIM micrograph of superplastic specimens deformed to $660 \%$ deformation. (a) shows an OIM in which the quality factor is depicted; (b) both low angle and high angle boundaries are drawn in as before as thin and thick lines respectively; (c) only the high angle lines are drawn.

was supported by a NASA Grant and the Center for Materials Research and Technology at Florida State University.

\section{References}

[1] B.P. Kashayap, A. Arieli, A.K. Mukherjee, J. Mater. Sci. 20 (1985) 2661.

[2] K. Morii, C. Hartig, H. Mecking, Y. Nakayama, G. Lutjering, in: J.S. Kallend, G. Gottstein (Eds.), Proc. 8th Int. Conf. on Texture of Materials, TMS, Warrendale, PA, 1988, pp. 991-996.

[3] A.K. Ghosh, C.H. Hamilton, Metall. Trans. 13A (1982) 733.

[4] P.N. Kalu, S. Hales, Textures in a biaxially superplastic formed Weldalite $^{\mathrm{TM}}$ 049, in: J.J. Jonas, T.R. Bieler, K.J. Bowman (Eds.), Advances in Hot Deformation Textures and Microstructures, TMS, Warrendale, 1994, pp. 385-395.

[5] A.K. Mukherjee, Ann. Rev. Mater. Sci. 9 (1979) 191-217.
[6] M.F. Ashby, R.A. Verrall, Acta Metall. Mater. 21 (1973) 149163.

[7] H. Gudmundsson, D. Brooks, J.A. Wert, Acta Metall. Mater. 39 (1991) 19-35

[8] T.G. Langdon, Mater. Sci. Eng. A137 (1991) 1-11.

[9] J.A. Venebles, C.J. Harland, Philos. Mag. 27 (1973) 1193-1200.

[10] K.Z. Baba-Kishi, D.J. Dingley, Scanning 11 (1989) 305-312.

[11] S.I. Wright, B.L. Adams, K. Kunze, Mater. Sci. Eng. A160 (1993) 229-240.

[12] Brent L. Adams, Mater. Sci. Eng. A166 (1993) 59-66.

[13] B.L. Adams, S.I. Wright, K. Kunze, Metall. Trans. 24A (1993) 819-831.

[14] V. Randle, Microtexture Determination and its Applications, Institute of Materials, 1992.

[15] D. Hull, D.J. Bacon, Introduction to dislocations, 3rd ed., Int. Series on Materials Science and Technology, Pergamon Press, Oxford, 1984.

[16] A. Ball, M.M. Hutchinson, Met. Sci. J. 3 (1969) 1.

[17] J.R. Spring, W.D. Nix, Acta Metall. Mater. 26 (1978) 13891398. 